Pacific Journal of Mathematics

A CHARACTERIZATION OF ANALYTICALLY UNRAMIFIED 


\title{
A CHARACTERIZATION OF ANALYTICALLY UNRAMIFIED SEMI-LOCAL RINGS AND APPLICATIONS
}

\author{
L. J. RATLIFF, JR.
}

It is proved that a semi-local ring $R$ is analytically unramified if and only if $R$ is a subspace of a ring which is isomorphic to a finite direct sum of semi-local Dedekind domains. Applying this, it is proved that a local domain $R$ is analytically irreducible if and only if $R$ is a subspace of a local Dedekind domain, and this is true if and only if $R$ is a subspace of every local domain which dominates $R$ and which satisfies the altitude formula relative to $R$. A final application proves that an analytically unramified local domain is unmixed if and only if it satisfies the altitude formula.

All rings in this article are commutative rings with a unit, and the terminology is in general the same as that in [2]. In particular a ring $R^{*}$ dominates a ring $R$ in case $R$ is a subring of $R^{*}$, each maximal ideal in $R^{*}$ contracts to a maximal ideal in $R$, and each proper ideal in $R$ is contained in a maximal ideal in $R^{*}$. A semi-local (Noetherian) ring $R$ is a subspace of a semi-local ring $R^{*}$ in case $R^{*}$ dominates $R$ and $R$ is a subspace of $R^{*}$ for the natural (Jacobson radical) topologies. A Dedekind domain is an integrally closed Noetherian domain of altitude one.

In $\S 2$ it is proved that a semi-local ring $R$ is analytically unramified if and only if $R$ is a subspace of a ring which is isomorphic to a finite direct sum of semi-local Dedekind domains (Theorem 2.1). When $R$ is analytically unramified, Theorem 2.2 associates with each ideal $B$ contained in the Jacobson radical $J$ of $R$ a ring $W$ such that (1) $W$ is a quotient ring of a finitely generated ring over $R$, and (2) $W$ is isomorphic to a finite direct sum of semi-local Dedekind domains. Further, if $\operatorname{Rad} B=J$, then (3) $R$ is a subspace of $W$, and (4) if $N$ is a maximal ideal in $W$, then $\operatorname{trd}(W / N) /(R / N \cap R)$ is equal to the depth of one (uniquely determined by $N$ ) of the prime divisors of zero in the completion of $R$ (Proposition 2.8). To prove Theorem 2.2 a number of preliminary lemmas are needed and among these results Lemmas 2.3 and 2.4 are of some interest in themselves although they follow quite readily from known results. Essentially the method of proof of Theorem 2.2 is a combination of the methods used by Rees in $[5,6,7,8]$ to prove the Valuation Theorem.

Applications of Theorem 2.2 are given in $\S 3,4$ and 5. Theorem 3.5 and Theorem 3.7 characterize the intersection of $W$ and the total 
quotient ring of $R$ under the additional assumption that $B$ is not contained in any of the prime divisors of zero in $R$. This ring also has the properties (1), (2), (3), and a variant of (4) listed above.

In $\S 4$ it is proved that a local domain $(R, M)$ is analytically irreducible if and only if $R$ is a subspace of a local Dedekind domain (Theorem 4.1). When $R$ is analytically irreducible, Theorem 4.2 associates with each $M$-primary ideal $B$ a finite number of local Dedekind domains which have properties (1), (3), and (4) above. Theorem 4.5 generalizes Theorem 4.1 and states that $R$ is analytically irreducible if and only if $R$ is a subspace of every local domain $(S, N)$ which dominates $R$ and is such that altitude $S+\operatorname{trd}(S / N) /(R / M)=$ altitude $R+\operatorname{trd} S / R<\infty$. Finally in $\S 5$, a special case of Theorem 5.2 proves that an analytically unramified local domain satisfies the altitude formula if and only if it is unmixed.

2. A characterization of analytically unramified semi-local rings. The following notation is fixed throughout this section:

$R$ is a semi-local ring with maximal ideals $M_{1}, \cdots, M_{h}, J=\bigcap_{1}^{h} M_{i}$ is the Jacobson radical of $R$, and $q_{1}, \cdots, q_{g}$ are the prime divisors of zero in $R$. (The numbers $h$ and $g$ are fixed.)

Also, the following two notational conventions will be used throughout this paper:

(1) If $B=\left(b_{1}, \cdots, b_{m}\right) A$ is an ideal in a ring $A$, then $\mathscr{R}(A, B)$ will denote the Rees ring $A\left[t b_{1}, \cdots, t b_{m}, u\right]$ ( $t$ an indeterminant, $u=1 / t$ ) of $A$ with respect to $B .(\mathscr{R}(A,(0))=A[u]$, and $\mathscr{R}(A, A)=A[t, u])$; and

(2) If $A$ is a ring, then $A^{\prime}$ will denote the integral closure of $A$ in its total quotient ring.

THEOREM 2.1. $R$ is analytically unramified if and only if $R$ is a subspace of a ring which is isomorphic to a direct sum of a finite number of semi-local Dedekind domains.

Proof. It is well known that a ring which is isomorphic to a direct sum of a finite number of semi-local Dedekind domains is analytically unramified. Hence, if $R$ is a subspace of such a ring, then $R$ is analytically unramified. The converse follows from the following.

THEOREM 2.2. Let $R$ be analytically unramified, let $B \subseteq J$ be an ideal in $R$, let $\mathscr{R}=\mathscr{R}(R, B)$, and let $S$ be the complement in

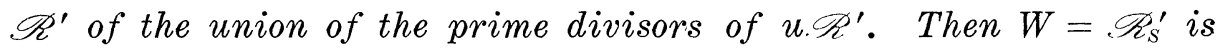
isomorphic to a direct sum of $g$ semi-local Dedekind domains. If $J$ is the radical of $B$, then $R$ is a subspace of $W$. 
To prove Theorem 2.2 some preliminary results are needed, so the proof of this theorem will be given following Lemma 2.6 below.

LEMMA 2.3. $R$ is analytically unramified if and only if $\cap_{1}^{g} q_{i}=$ (0) and $R / q_{i}$ is analytically unramified $(i=1, \cdots, g)$.

Proof. Let $R^{*}$ be the completion of $R$. If $R$ is analytically unramified, then clearly $(0)=\bigcap_{1}^{g} q_{i}$. To prove that each $R / q_{i}$ is analytically unramified is equivalent to proving that each $q_{i} R^{*}$ is an intersection of prime ideals, since $R^{*} / q_{i} R^{*}$ is isomorphic to the completion of $R / q_{i}$. Therefore, fix $i$ and let $P$ be a prime divisor of $q_{i} R^{*}$. Since $q_{i}$ is a prime divisor of zero in $R$ and since $P$ is a prime divisor of $q_{i} R^{*}$, it follows from [2, (18.11)] that $P$ is a prime divisor of zero in $R^{*}$. Hence, since $R$ is analytically unramified, $P$ is the $P$-primary component of $q_{i} R^{*}$. Therefore each $R / q_{i}$ is analytically unramified. Conversely, if each $q_{i} R^{*}$ is an intersection of prime ideals and $(0)=$ $\bigcap_{1}^{g} q_{i}$, then $(0) R^{*}=\left(\bigcap_{1}^{g} q_{i}\right) R^{*}=\bigcap_{1}^{g}\left(q_{i} R^{*}\right)$ is an intersection of prime ideals. Hence, from the uniqueness theorems on the primary decomposition of an ideal, $R$ is analytically unramified.

It is known [1, p. 365] that if an integral domain $A$ is a finite separable extension of an analytically unramified semi-local domain, then $A^{\prime}$ is a finite $A$-module and $A_{P}$ is analytically unramified for every prime ideal $P$ in $A$. This result is used in the proof of the following lemma.

Lemma 2.4. Let $\mathscr{R}$ be a finitely generated extension ring of $R$. Assume: (1) the zero ideal in $\mathscr{R}$ is an intersection of prime ideals $q_{i}^{*}$; (2) each $q_{i}^{*} \cap R$ is a prime divisor of zero in $R$; (3) each $\mathscr{R} / q_{i}^{*}$ is separable over $R / q_{i}^{*} \cap R$; and (4) $R$ is analytically unramified. Then the integral closure $\mathscr{R}^{\prime}$ of $\mathscr{R}$ is a finite $\mathscr{R}$-module and $\mathscr{R}_{S}$ is analytically unramified, where $S$ is the complement in $\mathscr{R}$ of the union of a finite number of prime ideals in $\mathscr{R}$.

Proof. Let $\mathscr{T}$ be the total quotient ring of $\mathscr{R}$. Then by (1) $\mathscr{T}$ can be considered to be the (finite, since $\mathscr{R}$ is Noetherian) direct sum of the fields $\mathscr{T} / q_{i}^{*} \mathscr{T}$ and then $D=\bigoplus \mathscr{R} / q_{i}^{*}$ is a finite $\mathscr{R}$-module such that $\mathscr{R} \cong D \cong \mathscr{T}$. Therefore $\mathscr{R} \cong D \subseteq \mathscr{R}^{\prime}$ and $\mathscr{R}^{\prime}=\bigoplus\left(\mathscr{R} / q_{i}^{*}\right)^{\prime}$. By (2), (4), and Lemma $2.3, R / q_{i}^{*} \cap R$ is an analytically unramified semi-local domain, and by (3) $\mathscr{R} / q_{i}^{*}$ is a finite separable extension of $R / q_{i}^{*} \cap R$, hence $\left(\mathscr{R} / q_{i}^{*}\right)^{\prime}$ is a finite $\mathscr{R} / q_{i}^{*}$-module [1]. Therefore $\mathscr{R}^{\prime}$ is a finite $D$-module, and hence $\mathscr{R}^{\prime}$ is a finite $\mathscr{R}$-module. Let $S$ be the complement in $\mathscr{R}$ of the union of a finite number of prime ideals in $\mathscr{R}$. To prove $\mathscr{R}_{S}$ is analytically unramified it is sufficient to prove that $\mathscr{R}_{P}$ is analytically unramified, for every 
prime ideal $P$ in $\mathscr{R}$ such that $P \mathscr{R}_{S}$ is maximal [2, (36.7)]. Therefore, let $K$ be the kernel of the natural homomorphism from $\mathscr{R}$ into $\mathscr{R}_{P}$, say $K=\bigcap_{1}^{s} q_{i}^{*}$. For each $i=1, \cdots, s, \mathscr{R}_{P} / q_{i}^{*} \mathscr{R}_{P}$ is a quotient ring of $\mathscr{R} / q_{i}^{*}$ and $\mathscr{R} / q_{i}^{*}$ is separably generated over $R / q_{i}^{*} \cap R$. Therefore $\mathscr{R}_{P} / q_{i}^{*} \mathscr{R}_{P}$ is analytically unramified [1], hence $\mathscr{R}_{P}$ is analytically unramified (Lemma 2.3).

REMARK 2.5. Let $R$ and $\mathscr{R}$ be as in Lemma 2.4 , let $p_{1}, \cdots, p_{n}$ be the prime divisors of zero in $\mathscr{R}^{\prime}$, and let $b$ be a nonunit in $\mathscr{R}^{\prime}$. Let $S$ be the complement in $\mathscr{R}^{\prime}$ of the union of the prime divisors of $b \mathscr{R}^{\prime}$, and assume that $b \in p_{i}$ if and only if $i \leqq k$, and $p_{i}+b \mathscr{R}^{\prime} \neq \mathscr{R}^{\prime}$ if and only if $i \leqq k+r$. Then $\mathscr{R}_{S}^{\prime}$ is isomorphic to a direct sum of $k$ fields and $r$ semi-local Dedekind domains.

Proof. $\mathscr{R}^{\prime}$ is Noetherian, since $\mathscr{R}^{\prime}$ is a finite $\mathscr{R}$-module. Therefore a prime ideal $P$ is a prime divisor of an ideal $B$ in $\mathscr{R}^{\prime}$ if and only if $P \mathscr{R}_{P}^{\prime}$ is a prime divisor of $B \mathscr{R}_{P}^{\prime}$. Since $\mathscr{R}^{\prime}$ is integrally closed, each prime ideal $P$ in $\mathscr{R}^{\prime}$ contains exactly one prime divisor $p_{i}$ of zero, so $\mathscr{R}_{P}^{\prime}$ is a quotient ring of the integrally closed Noetherian domain $\mathscr{R}^{\prime} / p_{i}$. The proof is completed by using the primary decomposition of a principal ideal in an integrally closed Noetherian domain and the fact that $\mathscr{R}^{\prime} \cong \bigoplus \mathscr{R}^{\prime} / p_{i}$.

Lemma 2.6. Let $A$ be a Noetherian ring, and let $b$ be a nonunit nonzero-divisor in $A$. If $A^{\prime}$ is a finite A-module, then there is a positive integer $n$ (depending on $b$ ) such that $b^{n+i} A^{\prime} \cap A \subseteq b^{i} A$, for all $i \geqq 1$.

Proof. If $A^{\prime}$ is a finite $A$-module, then, since $A$ is Noetherian, $D=A^{\prime} \cap A[1 / b]$ is a finite $A$-module. Since every element in $D$ may be written in the form $r / b^{k}$, where $r \in b^{k} A^{\prime} \cap A$, it follows that $b^{n} D \subseteq A$, for some $n \geqq 1$. Therefore, if $c \in b^{n+i} A^{\prime} \cap A$, then $c / b^{n+i} \in D$, hence $c \in b^{i} A$.

From the definition of the Rees ring $\mathscr{R}=\mathscr{R}(R, B)$ of $R$ with respect to $B$ it follows that $\mathscr{R}$ is a graded Noetherian ring (the homogeneous elements of degree $k$ being the elements $t^{k} b$, where $b \in B^{k}$, if $k>0$, and $b \in R$, if $k \leqq 0$ ), $u$ is not a zero divisor in $\mathscr{R}$, and $u^{i} \mathscr{R} \cap R=B^{i}$, for all $i \geqq 1$. Also, it is known [9, Lemma 1.1] that if $C$ is an ideal in $R$ and $C^{*}=C R[t, u] \cap \mathscr{R}$, then

$$
\mathscr{R} / C^{*} \cong \mathscr{R}(R / C, B+C / C) \text {. }
$$

This implies that if $q$ is a prime divisor of zero in $R$ and $\mathscr{T}$ is the total quotient ring of $\mathscr{R}$, then $\mathscr{R} / q \mathscr{T} \cap \mathscr{R} \cong \mathscr{R}(R / q, B+q / q)$. 
These facts are used in the following.

Proof of Theorem 2.2. Let $\mathscr{T}$ be the total quotient ring of $\mathscr{R}$. Since $\mathscr{T}$ is the total quotient ring of $R[t]$, and since $\mathscr{R} / q_{i} \mathscr{T} \cap \mathscr{R}$ is isomorphic to $\mathscr{R}_{i}=\mathscr{R}\left(R / q_{i}, B+q_{i} / q_{i}\right)(i=1, \cdots, g)$ [9], $\mathscr{R}^{\prime}$ is a finite $\mathscr{R}$-module (Lemma 2.4). Further, $\mathscr{R}^{\prime} \cong \bigoplus_{1}^{g}\left(\mathscr{R}_{i}\right)^{\prime}$, since $\mathscr{R}_{i} \cong$ $\mathscr{R} / q_{i} \mathscr{T} \cap \mathscr{R}$. Hence $u \mathscr{R}^{\prime}+\left(q_{i} \mathscr{T} \cap \mathscr{R}^{\prime}\right) \neq \mathscr{R}^{\prime}(i=1, \cdots, g)$, since $B \subseteq J$ implies $u$ is a nonunit in $\mathscr{R}_{i}{ }^{\prime}$. Therefore $W=\mathscr{R}_{S}^{\prime}$ is isomorphic to a direct sum of $g$ semi-local Dedekind domains (Remark 2.5), and $W \subset \mathscr{T}$. Further, since $\mathscr{R}^{\prime}$ is a finite $\mathscr{R}$-module, there is a positive integer $n$ such that, for all $i \geqq 1, u^{n+i} \mathscr{R}^{\prime} \cap \mathscr{R} \subseteq u^{i} \mathscr{R}$ (Lemma 2.6). Therefore

$$
u^{n+i} W \cap R=\left(\left(u^{n+i} W \cap \mathscr{R}^{\prime}\right) \cap \mathscr{R}\right) \cap R \subseteq u^{i} \mathscr{R} \cap R=B^{i} \leqq u^{i} W \cap R,
$$

for all $i \geqq 1$. Now the Jacobson radical of $W$ is the radical of $u W$, and the contraction of the radical of an ideal is the radical of the contraction of the ideal. Hence, if $J$ is the radical of $B$, then $R$ is a subspace of $W$.

COROLlary 2.7. With the same notation as Theorem 2.2, let $U$ be the complement in $R$ of the union of some of the minimal prime divisors of $B$. Then $R_{U}$ is a subspace of $W_{L^{*}}$.

Proof. Since $R$ is analytically unramified, $R_{U}$ is analytically unramified (Lemma 2.4). Let $K$ be the kernel of the natural homomorphism from $R$ into $R_{U}$. Then $K^{*}=K \mathscr{T} \cap \mathscr{R}$ is the kernel of the natural homomorphism from $\mathscr{R}$ into $\mathscr{R}_{U}$. Since $\mathscr{R} / K^{*}$ is isomorphic to $\mathscr{R}(R / K, B+K / K)$, it is easily seen that

$$
\mathscr{R}(R, B)_{U} \cong R^{*}=\mathscr{R}\left(R_{U}, B R_{U}\right),
$$

and from this it follows that $W_{U} \cong\left(R^{* \prime}\right)_{S^{\prime}}$, where $S^{\prime}$ is the complement in $R^{* \prime}$ of the union of the prime divisors of $u R^{* \prime}$. Therefore $R_{U}$ is a subspace of $W_{U}$ (Theorem 2.2).

REMARK. With the same notation as Theorem 2.2, assume that $B \nsubseteq J, B \subseteq q_{i}$ if and only if $i \leqq k$, and $B+q_{i} \neq R$ if and only if $i \leqq k+r$. Then

$$
\mathscr{R} / q_{i} \mathscr{T} \cap \mathscr{R} \cong \mathscr{R}_{i}=\mathscr{R}\left(R / q_{i}, B+q_{i} / q_{i}\right)
$$

implies $\mathscr{R}_{i}=R / q_{i}[t, u](i=k+r+1, \cdots, g)$, and $\mathscr{R}_{i}=R / q_{i}[u](i=$ $1, \cdots, k)$. Therefore $W=\mathscr{R}_{S}^{\prime}$ is isomorphic to a direct sum of $k$ local Dedekind domains and $r$ semi-local Dedekind domains.

An integral domain $A$ satisfies the altitude formula in case it is 
true that for every finitely generated integral domain $D$ over $A$, if $P$ is a prime ideal in $D$, then

$$
\text { height } P+\operatorname{trd}(D / P) /(A / P \cap A)=\text { height } P \cap A+\operatorname{trd} D / A
$$

(where $\operatorname{trd}(E / F)$ denotes the transcendence degree of the integral domain $E$ over its subdomain $F$ ). It is known [3, Corollary 2.9] that an analytically irreducible local domain satisfies the altitude formula.

Proposition 2.8. With the notation of Theorem 2.2 let $\operatorname{Rad} B=J$ so that the completion $R^{*}$ of $R$ is a subring of the completion $W^{*}$ of $W$. Let $Q$ be a prime divisor of $u \mathscr{R}^{\prime}$, let $p^{*}$ be the prime divisor of zero in $W^{*}$ which is contained in $Q W^{*}$, and let $p=p^{*} \cap R^{*}$. Then $p$ is a prime divisor of zero in $R^{*}$ and

$$
\operatorname{depth} Q=\operatorname{trd}\left(\mathscr{R}^{\prime} / Q\right) /(R / Q \cap R)=\operatorname{depth} p .
$$

Proof. Let $\mathscr{R}^{*}=\mathscr{R}\left(R^{*}, B R^{*}\right)$. Then $\mathscr{R}^{\prime}$ is a subring of $\mathscr{R}^{* \prime}$. Since $W^{*}$ is isomorphic to a direct sum of local Dedekind domains and $R^{*}$ is a subring of $W^{*}$ (Theorem 2.2), it follows that $\mathscr{R}^{* \prime}$ is a subring of $W^{*}$. Since $Q W$ is a maximal ideal in $W, Q W^{*}$ contains only one prime divisor $p^{*}$ of zero in $W^{*}$ and $p^{\prime}=p^{*} \cap \mathscr{R}^{* \prime} \subset Q^{*}=$ $Q W^{*} \cap \mathscr{R}^{* \prime}$ (since $u \notin p^{*}$ ). To prove that $p=p^{*} \cap R^{*}$ is a prime divisor of zero in $R^{*}$ it suffices to prove that $p^{\prime}$ is a prime divisor of zero in $\mathscr{R}^{* \prime}$. Now $Q^{*}$ contains a (height one) prime divisor $N$ of $u \mathscr{R}^{* \prime}$, and then $N \cap \mathscr{R}^{\prime}=Q^{*} \cap \mathscr{R}^{\prime}=Q$. Also $\mathscr{R}^{* \prime} / N$ is integrally dependent on $\mathscr{R}^{*} / N \cap \mathscr{R}^{*}$, and $\mathscr{R}^{*} / N \cap \mathscr{R}^{*}=\mathscr{R} / N \cap \mathscr{R}$ (since $u \in N$ and $\operatorname{Rad} B=J$ imply $\left.R^{*} / N \cap R^{*}=R / N \cap R\right)$. Hence, since $\mathscr{R}^{* \prime}\left|N \supseteqq \mathscr{R}^{\prime}\right| N \cap \mathscr{R}^{\prime} \supseteqq \mathscr{R} \mid N \cap \mathscr{R}$ and $\left(Q^{*} / N\right) \cap\left(\mathscr{R}^{\prime} / N \cap \mathscr{R}^{\prime}\right)=(0)$, it follows that $Q^{*}=N$, so $p^{\prime}$ is a prime divisor of zero in $\mathscr{R}^{* \prime}$. Since $\mathscr{R}^{* \prime} / Q^{*}$ is integrally dependent on $\mathscr{R}^{\prime} / Q$ and $\mathscr{R}^{\prime} / Q$ is a finitely generated integral domain over the field $R / Q \cap R=R^{*} / Q^{*} \cap R^{*}$, it follows that

$$
\operatorname{trd}\left(\mathscr{R}^{* \prime} / Q^{*}\right) /\left(R^{*} / Q^{*} \cap R^{*}\right)=\operatorname{trd}\left(\mathscr{R}^{\prime} / Q\right) /(R / Q \cap R)=\operatorname{depth} Q .
$$

Finally, $R^{*} / p$ satisfies the altitude formula [3] and

$$
\mathscr{R}^{* \prime} / p^{\prime} \cong \mathscr{R}\left(R^{*} / p, B R^{*}+p / p\right)^{\prime} .
$$

Hence $\operatorname{trd}\left(\mathscr{R}^{* \prime} / Q^{*}\right) /\left(R^{*} / Q^{*} \cap R^{*}\right)=$ altitude $R^{*} / p=$ depth $p$, since height $Q^{*} / p^{\prime}=\operatorname{trd}\left(\mathscr{R}^{* \prime} / p^{\prime}\right) /\left(R^{*} / p\right)=1$.

REMARK 2.9. With the notation of Proposition $2.8 Q W^{*} \cap \mathscr{R}^{* \prime}$ is a (height one) prime divisor of $u \cdot \mathscr{R}^{* \prime}$. Also, if a prime divisor $p$ of zero in $R^{*}$ is given, then there is a prime divisor $Q$ of $u \mathscr{R}^{\prime}$ such. 
that the prime divisor $p^{*}$ of zero in $W^{*}$ which is contained in $Q W^{*}$ is such that $p^{*} \cap R^{*}=p$ (since the radical of an ideal contracts to the radical of the contraction of the ideal).

3. The ring $W \cap T$. Let $R, B \subseteq J$, and $W$ be as in Theorem 2.2 , and let $T$ be the total quotient ring of $R$. It is known [2, (33.7)] that if $V$ is a local Dedekind domain and $F$ is a subfield of the quotient field of $V$, then $V \cap F$ is either a local Dedekind domain or $F$. This implies that if $B$ is contained in exactly $k$ of the ideals $q_{i}$, then $W \cap T$ is isomorphic to a direct sum of $k$ fields and $g-k$ semilocal Dedekind domains. Under the assumption that $B \nsubseteq q_{i}(i=1, \cdots, g)$, Theorems 3.5 and 3.7 below describe $W \cap T$ as the quotient ring of a finitely generated ring over $R$ and Theorem 3.10 gives a related result. To prove these theorems the following notation will be fixed throughout this section:

$R$ is an analytically unramified semi-local ring with maximal ideals $M_{1}, \cdots, M_{h}, J$ is the Jacobson radical of $R$, and $q_{1}, \cdots, q_{g}$ are the prime divisors of zero in $R . \quad B=\left(b_{1}, \cdots, b_{m}\right) R$ is contained in $J$ and $B \nsubseteq q_{i}(i=1, \cdots, g)$ (so height $\left.M_{i} \geqq 1 \quad(i=1, \cdots, h)\right)$. Let $\mathscr{R}=$ $\mathscr{R}(R, B)$, let $Q_{1}, \cdots, Q_{s}$ be the prime divisors of $u \mathscr{R}^{\prime}$, and let $P_{i}=$ $Q_{i} \cap \mathscr{R}$ and $P_{i}=P_{i} \cap R(i=1, \cdots, s)$. (There may be less than $s$ distinct $p_{i}$ or $p_{i}$.) Let $T(\mathscr{S})$ be the total quotient ring of $R(\mathscr{R})$, let $S=\mathscr{R}^{\prime} \sim \bigcup_{1}^{s} Q_{i}$, and let $W=\mathscr{R}_{S}^{\prime}$.

REMARK 3.1. Since $B \subseteq J, W$ is isomorphic to a direct sum of $g$ semi-local Dedekind domains (Theorem 2.2). Therefore, each $q_{i} \mathscr{T} \cap \mathscr{R}^{\prime}$ is contained in at least one $Q_{j}$, so $q_{i} \mathscr{G} \cap \mathscr{R} \subset P_{j}$. Hence each $q_{i}$ is contained in at least one $p_{j}$ and the containment is proper, since $B \nsubseteq q_{i}(i=1, \cdots, g)$ and $B \cong p_{j}(j=1, \cdots, s)$.

\section{Lemma 3.2. (1) $\operatorname{trd}\left(\mathscr{R}^{\prime} / Q_{i}\right) /\left(R / p_{i}\right) \geqq 1(i=1, \cdots, s)$.}

(2) There exists a positive integer $k$ (depending on $B$ ) and an element $b \in B^{k}$ such that $t^{k} b \in p_{i}(i=1, \cdots, s)$.

Proof. (1) Fix $i$ and let $Q=Q_{i}, P=P_{i}$, and $p=p_{i}$. Let $q \mathscr{S} \cap \mathscr{R}^{\prime}$ be the minimal prime ideal contained in $Q$. Since $\mathscr{R}^{\prime} / Q$ is isomorphic to a factor ring of $\mathscr{R}(R / q, B+q / q)^{\prime}$ [9], to prove (1) it may be assumed that $R$ is an integral domain. Let $V^{*}=\mathscr{R}_{Q}^{\prime}$, let $N^{*}=Q V^{*}$, and let $F$ be the quotient field of $R$. Since $V^{*}$ is a local Dedekind domain and $N=N^{*} \cap F \supseteqq p \neq(0)$ (Remark 3.1), $V=V^{*} \cap F$ is a local Dedekind domain [2, (33.7)]. Therefore $b_{j} V=B V$, for some $j=1, \cdots, m$, so $R\left[b_{1} / b_{j}, \cdots, b_{m} / b_{j}\right] \leqq V$. Thus $D=V\left[t b_{j}, u\right] \supseteqq \mathscr{R}$, so $\mathscr{R}_{Q}^{\prime}=V^{*}=D_{N^{*} \cap D^{\prime}}^{\prime}$. Now $D^{\prime}$ is a finite $D$-module [1] and $V$ satisfies 
the altitude formula [3], hence $\operatorname{trd}\left(D^{\prime} / N^{*} \cap D^{\prime}\right) /(V / N)=1$. Since $D^{\prime} / N^{*} \cap D^{\prime}$ and $\mathscr{R}^{\prime} / Q$ have the same quotient field, and since $V / N \supseteqq R / p$, (1) is proved. It now follows with the above notation that:

(3.1) In the general case (when $R$ is not assumed to be an integral domain) $t b_{j} \notin Q$, and when $R$ is an integral domain $V^{*}=$ $V[t b]_{N V[t b]}$, for each $b \in B$ such that $b V=B V$.

Since $\mathscr{R}^{\prime}$ is isomorphic to a direct sum of integrally closed Noetherian domains and each $Q_{i}$ is a prime divisor of $u \mathscr{R}^{\prime}$, it follows from [2, (33.11)] that each $P_{i}$ is a prime divisor of $u \mathscr{R}$. Hence, since $\mathscr{R}$ is a graded ring, each $P_{i}$ is a homogeneous ideal which contains all elements of negative degree. Also $B^{*}=\left(t b_{1}, \cdots, t b_{m}\right) \mathscr{R}$ is a homogeneous ideal in $\mathscr{R}$. Therefore to prove (2) it is sufficient to prove that $B^{*} \nsubseteq P_{i}(i=1, \cdots, s)$, and this follows from (3.1).

REMARK 3.3. Since each $q_{j} \mathscr{T} \cap \mathscr{R}$ is contained in some $P_{i}$, if $b \in B^{k}$ is such that $t^{k} b \notin P_{i}(i=1, \cdots, s)$, then $b$ is not a zero-divisor in $R$. Also, if $R / M_{j}$ is an infinite field $(j=1, \cdots, h)$, then it can be shown that there is an element $b \in B$ such that $t b \notin P_{i}(i=1, \cdots, s)$.

If $B$ is an ideal in a ring $A$, then the set $B_{a}$ of elements $x$ in $A$ such that $x$ satisfies an equation of the form

$$
x^{n}+b_{1} x^{n-1}+\cdots+b_{n}=0,
$$

where $b_{i} \in B^{i}(i=1, \cdots, n)$, is the integral closure of $B$ in $A$. Clearly $B \sqsubseteq B_{a}$.

LEMMA 3.4. Let $b$ be a nonzero-divisor in $\left(B^{k}\right)_{a}$, where $k \geqq 1$, let $C=\mathscr{R}^{\prime}\left[1 / t^{k} b\right]$, and let $A=R\left[\beta_{1} / b, \cdots, \beta_{r} / b\right]$ where $B^{k}=\left(\beta_{1}, \cdots, \beta_{r}\right) R$. Then there is a one to one correspondence between the prime divisors $Q$ of $u C$ and the prime divisors $\mathscr{P}$ of $b A^{\prime}$ such that if $\mathscr{P}$ and $Q$ correspond, then $\mathscr{P}=Q \cap A^{\prime}$ and $Q=Q_{i} C$, for some $i=1, \cdots, s$.

Proof. Let $A^{*}=A^{\prime}\left[t^{k} b, 1 / t^{k} b\right]$. Then $A^{*}$ is an integrally closed Noetherian ring since $A^{\prime}$ is (Lemma 2.4). An equation of integral dependence of $b$ on $B^{k}$ shows that $t^{k} b \in \mathscr{R}^{\prime}$, so $C$ is a quotient ring of $\mathscr{R}^{\prime}$, hence $C$ is integrally closed. Therefore, since the elements $t^{k} \beta_{i}(i=1, \cdots, r)$ are in $\mathscr{R}, A^{*} \leqq C$. Further, $C$ is integrally dependent on $A^{*}$, since $\mathscr{R}^{\prime}$ is integrally dependent on

$$
R\left[t^{k} \beta_{1}, \cdots, t^{k} \beta_{r}, u^{k}\right] \subseteq A^{*} .
$$

(If $k=1$, then $C=A^{*}$.) Since $C$ is integrally dependent on $A^{*}$ and $b A^{*}=u^{k} A^{*}$, it follows that $b A^{\prime}=A^{\prime}$ if and only if $u C=C$, and if 
$\mathscr{P}$ is a prime divisor of $b A^{\prime}$, then there is a prime divisor $Q_{i} C$ (for some $i=1, \cdots, s)$ of $u C$ such that $Q_{i} C \cap A^{*}=\mathscr{P} A^{*}$. Now let $Q$ be a prime divisor of $u C$, and let $q \mathscr{T} \cap C$ be the minimal prime ideal contained in $Q$. Then $q \mathscr{T} \cap A^{*}$ is the minimal prime ideal contained in $Q \cap A^{*}$. Hence, since $A^{*} / q \mathscr{T} \cap A^{*}$ is integrally closed, height $Q \cap A^{*}=1[2,(10.14)]$, so $Q \cap A^{\prime}$ is a prime divisor of $b A^{\prime}$. Finally, let $V^{*}=C_{Q}=\mathscr{R}_{Q}^{\prime} \mathscr{R}^{\prime}$, let $N^{*}=Q V^{*}$, and let $F$ be the quotient field of $R / q$. Then $V^{*} \supseteqq A^{\prime} / q T \cap A^{\prime}$ and, since height $Q \cap A^{\prime}=1$, height $\left(N^{*} \cap\left(A^{\prime} / q T \cap A^{\prime}\right)\right)=1$. Therefore (3.1) implies that distinct prime divisors of $u C$ which contain $q \mathscr{T} \cap C$ must contract to distinct prime divisors of $b A^{\prime}$. Hence distinct prime divisors of $u C$ contract to distinct prime divisors of $b A^{\prime}$.

THEOREM 3.5. With the fixed notation of this section, let

$$
b \in B^{k}=\left(\beta_{1}, \cdots, \beta_{r}\right) R
$$

be such that $t^{k} b \notin P_{i}(i=1, \cdots, s)$, let $A=R\left[\beta_{1} / b, \cdots, \beta_{r} / b\right]$, and let $U$ be the complement in $A^{\prime}$ of the union of the prime divisors of $b A^{\prime}$. Then $A_{U}^{\prime}=W \cap T$. If $J$ is the radical of $B$, then $R$ is a subspace of $A_{U}^{\prime}$.

Proof. The existence of $t^{k} b \notin P_{i}(i=1, \cdots, s)$ is given by Lemma 3.2, and then Remark 3.3 shows that Lemma 3.4 is applicable for such $b \in B^{k}$. Therefore by Lemma 3.4 the ideals $\mathscr{P}_{i}=Q_{i} \mathscr{R}^{\prime}\left[1 / t^{k} b\right] \cap A^{\prime}$ are distinct and are the prime divisors of $b A^{\prime}$, so $A_{U}^{\prime} \subseteq \mathscr{R}^{\prime}\left[1 / t^{k} b\right]_{U}=W$, hence $A_{U}^{\prime} \subseteq W \cap T$. Therefore for each $i=1, \cdots, g$,

$$
D_{i}=A_{U}^{\prime} / q_{i} T \cap A_{U}^{\prime} \subseteq E_{i}=(W \cap T) / q_{i} T \cap(W \cap T)
$$

and $E_{i}$ is contained in the quotient field $T / q_{i} T$ of $D_{i}$. Thus, since $A_{U}^{\prime} \cong \bigoplus_{1}^{g} D_{i}$ and $D_{i}$ is a semi-local Dedekind domain (Remark 2.5), and since $W \cap T$ is integrally closed in $T$, to prove that $A_{U}^{\prime}=W \cap T$ it is sufficient to prove that $D_{i}=E_{i}(i=1, \cdots, g)$. Since $D_{i}=\bigcap D_{i N}$, where $N$ runs over the maximal ideals in $D_{i}$, and since $D_{i N}$ is a maximal proper subring of its quotient field, $D_{i}=E_{i}$ if it is shown that for each maximal ideal $N$ in $D_{i}$ there is a prime ideal $N^{\prime}$ in $E_{i}$ such that $N^{\prime} \cap D_{i}=N$. This, for each $i=1, \cdots, g$, follows immediately on considering the ideals $Q_{j} W \cap(W \cap T)(j=1, \cdots, s)$. Finally, if $\operatorname{Rad} B=J$, then $R$ is a subspace of $W$ (Theorem 2.2), hence $R$ is a subspace of $A_{U}^{\prime}$.

Corollary 3.6. $A_{U}^{\prime}$ depends only on $B$. That is, $A_{U}^{\prime}$ is independent of the choice of $k$ and $b \in B^{k}$ such that $t^{k} b \notin P_{i}(i=1, \cdots, s)$.

Proof. This follows from $A_{U}^{\prime}=W \cap T$. 
REMARK. With the notation of Theorem 3.5 let $\operatorname{Rad} B=J$. Let $\mathscr{P}$ be a prime divisor of $b A^{\prime}$ and let $Q$ be the prime divisor of $u \mathscr{R}^{\prime}$ such that $Q W \cap A^{\prime}=\mathscr{P}$ (Lemma 3.4). Then it follows from (3.1) and Proposition 2.8 that

$$
\operatorname{depth} \mathscr{P}=\operatorname{trd}\left(A^{\prime} / \mathscr{P}\right) /(R / \mathscr{P} \cap R)=\operatorname{depth} Q-1 .
$$

Theorem 3.7 below shows in particular that $W \cap T$ is determined by any ideal between $B^{n}$ and $\left(B^{n}\right)_{a}$, where $n \geqq 1$.

Theorem 3.7. Let $R, B, t^{k} b, A, U$, and $T$ be as in Theorem 3.5. Let $R^{*}$ be a ring such that $R \cong R^{*} \cong R^{\prime}$, let $n \geqq 1$, let $B^{*}$ be an ideal in $R^{*}$ such that $B^{n} R^{*} \subseteq B^{*} \subseteq B^{n} A^{\prime} \cap R^{*}$, and let $\Sigma=\mathscr{R}\left(R^{*}, B^{*}\right)$. Then:

(1) $A^{\prime}=R^{*}\left[\gamma_{1} / b^{n}, \cdots, \gamma_{d} / b^{n}\right]^{\prime}$, where $B^{* k}=\left(\gamma_{1}, \cdots, \gamma_{d}\right) R^{*}$;

(2) $u \Sigma^{\prime}$ has exactly s prime divisors;

(3) $\Sigma_{S^{\prime}}^{\prime} \cap T=A_{U}^{\prime}$, where $S^{\prime}$ is the complement in $\Sigma^{\prime}$ of the union of the prime divisors of $u \Sigma^{\prime}$; and

(4) $u^{i} \Sigma^{\prime} \cap R^{*}=\left(B^{* i}\right)_{a}$ and $b^{n i} A^{\prime} \cap R^{*}=\left(B^{* k i}\right)_{a}$, for all $i \geqq 1$.

Proof. Since

$$
B^{k n} R^{*} \sqsubseteq B^{* k} \sqsubseteq B^{k n} A^{\prime} \cap R^{*}=b^{n} A^{\prime} \cap R^{*}
$$

and $A^{\prime}=R\left[c_{1} / b^{n}, \cdots, c_{e} / b^{n}\right]^{\prime}$, where $B^{k n}=\left(c_{1}, \cdots, c_{e}\right) R$, (1) is straightforward. To prove (2) it will first be shown that $u \mathscr{R}_{n}^{\prime}$ has exactly $s$ prime divisors, where $\mathscr{R}_{n}=\mathscr{R}\left(R, B^{n}\right)$. Now $\mathscr{R}_{n}^{\prime}\left[1 / t^{k} b^{n}\right] \supseteqq A^{\prime}$ (by (1)), so Lemma 3.4 (applied to $b^{n} \in\left(B^{n k}\right)_{a}$ ) and (1) show that there is a one to one correspondence between the prime divisors of $u \mathscr{R}_{n}^{\prime}\left[1 / t^{k} b^{n}\right]$ and $b^{n} A^{\prime}$. Therefore, since $b^{n} A^{\prime}$ and $b A^{\prime}$ have the same prime divisors and $b A^{\prime}$ has $s$ prime divisors (by the proof of Theorem 3.5), $u \mathscr{R}_{n}^{\prime}$ has at least $s$ prime divisors. Also, if $B^{n}=\left(d_{1}, \cdots, d_{f}\right) R$, then $\mathscr{R}_{n}$ is isomorphic to $D=R\left[t^{n} d_{1}, \cdots, t^{n} d_{f}, u^{n}\right]$ and $\mathscr{R}=\mathscr{R}(R, B)$ is integrally dependent on $D$. Hence, since $t^{k} b$ is not in any prime divisor of $u \mathscr{R}^{\prime}$, the integral dependence of $\mathscr{R}^{\prime}$ on $D^{\prime} \cong \mathscr{R}_{n}^{\prime}$ implies that $t^{k} b^{n}$ is not in any prime divisor of $u \mathscr{R}_{n}^{\prime}$. Therefore $u \mathscr{R}_{n}^{\prime}$ has exactly $s$ prime divisors. Now $\mathscr{R}_{n} \subseteq \Sigma$, so (2) holds if $\Sigma \subseteq \mathscr{R}_{n}^{\prime}$. For this it suffices to prove that if $x \in B^{*}$, then $t x \in \mathscr{R}_{n}^{\prime}$. Now $x \in B^{*}$ implies

$$
x^{k} \in B^{n k} A^{\prime}=b^{n} A^{\prime} \leqq b^{n} A^{\prime}\left[t^{k} b^{n}, 1 / t^{k} b^{n}\right] \leqq u^{k} \mathscr{R}_{n}^{\prime}\left[1 / t^{k} b^{n}\right] .
$$

Hence, since $t^{k} b^{n}$ is not in any prime divisor of $u \mathscr{R}_{n}^{\prime}$ and $x \in R^{\prime}$, $(t x)^{k} \in \mathscr{R}_{n}^{\prime}$. Thus, since $\mathscr{R}_{n}^{\prime}$ is integrally closed, $t x \in \mathscr{R}_{n}^{\prime}$, hence (2) holds. $R^{*}$ is analytically unramified since $R$ is. Therefore, (3) holds by (1), (2), and the proof of Theorem 3.5 , since 


$$
\Sigma^{\prime}=\mathscr{R}_{n}^{\prime}, \mathscr{R}_{n}^{\prime}\left[1 / t^{k} b^{n}\right] \supseteqq A^{\prime},
$$

and $t^{k} b^{n}$ is not in any prime divisor of $u \mathscr{R}_{n}^{\prime}$. For (4), if $x \in u^{i} \Sigma^{\prime} \cap R^{*}$, then $t^{i} x \in \Sigma^{\prime}$, so there are elements $f_{1}, \cdots, f_{j}$ in $\Sigma$ such that

$$
\left(t^{i} x\right)^{j}+f_{1}\left(t^{i} x\right)^{j-1}+\cdots+f_{j}=0 .
$$

Since $\Sigma$ is a graded subring of $R^{*}[t, u]$, and since each term on the left side of this equation is in $R^{*}[t, u]$, the definition of $\mathscr{R}\left(R^{*}, B^{*}\right)$ and the $j i$-th component show that $x \in\left(B^{* i}\right)_{a}$. If $x \in b^{n i} A^{\prime} \cap R^{*}$, then

$$
x \in b^{n i} A^{\prime}\left[t^{k} b^{n}, 1 / t^{k} b^{n}\right] \cap R^{*} \cong u^{k i} \Sigma^{\prime}\left[1 / t^{k} b^{n}\right] \cap \Sigma^{\prime} \cap R^{*} .
$$

Therefore, since $t^{k} b^{n}$ is not in any prime divisor of $u \Sigma^{\prime}, x \in u^{k i} \Sigma^{\prime} \cap R^{*}=$ $\left(B^{* k i}\right)_{a}$. The opposite inclusions follow readily by dividing an equation of integral dependence (of degree $j$ ) by $u^{i j}$ (by $b^{n i j}$ ) and using the definition of $\Sigma$ (using (1)).

Theorem 3.10 below gives a result which is related to Theorems 3.5, 3.7, and the "necessity" part of Theorem 4.1 below. Its proof is based on Lemma 3.8 below. The following facts will be used in the proof of Lemma 3.8.

Let $b$ be a nonzero-divisor in a Noetherian ring $A$. Then it is known [4, Lemma 1] that $(b A)_{a}=b A^{\prime} \cap A$, and an element $c \in A$ is in $(b A)_{a}$ if and only if $c / b \in A^{\prime}$. Also [4, Lemmas 2 and 4] $b A=(b A)_{a}$ if and only if $A_{p}$ is a local Dedekind domain, for each prime divisor $p$ of $b A$. Finally, if $A^{\prime}$ is isomorphic to a (finite) direct sum of Krull domains, then it follows from [2, (33.11)] that each prime divisor of $b A^{\prime}$ contracts in $A$ to a prime divisor of $b A$.

LEMMA 3.8. Let $Q_{1}, \cdots, Q_{v}$ be specified prime divisors of $u \mathscr{R}^{\prime}$ $(v \leqq s)$. Then there is an integer $n$ (depending on $B$ and $Q_{1}, \cdots, Q_{v}$ ) and a nonzero-divisor $c \in\left(B^{n}\right)_{a}$ such that $t^{n} c \in Q_{i}(i=1, \cdots, v)$ and $t^{n} c \notin Q_{i}(i=v+1, \cdots, s)$.

Proof. Let $b$ and $A$ be as in Theorem 3.5 and let $C=\mathscr{R}^{\prime}\left[1 / t^{k} b\right]$ so that $b A^{\prime}$ has the $s$ ideal $Q_{i} C \cap A^{\prime}$ as its prime divisors (Lemma 3.4). Let $D=A^{\prime} \cap A[1 / b]$. Then $D$ is Noetherian, since $A^{\prime}$ is a finite $A$-module (Lemma 2.4), and $D$ is the set of elements $x / b^{m}$, where $x \in b^{m} A^{\prime} \cap A$. By the definition of $D$ and the remarks preceding this lemma it follows that $b D=(b D)_{a}$, and then that the prime divisors of $b D$ are the $s$ height one prime ideals $\mathscr{P}_{i}=\left(Q_{i} C \cap A^{\prime}\right) \cap D$. Hence, since there are no containment relations among the $\mathscr{P}_{i}$, there is an element $x / b^{m}$ in $D$ which is in $\mathscr{P}_{i}$ if and only if $i \leqq v$, and it can be assumed that $x / b^{m}$ is not a zero-divisor. Then, by the definition of $A$, there is an integer $i$ and a nonzero-divisor $c \in B^{k i}$ such that $x / b^{m}=$ $c / b^{m+i}$. Since $A^{\prime} \subseteq C$ and $t^{k} b$ is a unit in $C, c / b^{m+i}$ and $c t^{m k+i k}$ generate 
the same ideal in $C$. Let $n=k(m+i)$. Then $c \in u^{n} C \cap \mathscr{R}^{\prime}=u^{n} \mathscr{R}^{\prime}$, hence $t^{n} c \in \mathscr{R}^{\prime}$ and $t^{n} c \in Q_{i} C \cap \mathscr{R}^{\prime}$ if and only if $i \leqq v$. Finally, $c \in u^{n} \mathscr{R}^{\prime} \cap R=\left(B^{n}\right)_{a}$ (Theorem 3.7).

COROLlaRY 3.9. There is an integer $n$ (depending on $B$ ) and $a$ nonzero-divisor $c \in\left(B^{n}\right)_{a}$ such that $c A^{\prime}$ is a primary ideal, where $B^{n}=\left(c_{1}, \cdots, c_{e}\right) R$ and $A=R\left[c_{1} / c, \cdots, c_{e} / c\right]$.

Proof. This follows immediately from Lemma 3.8 and 3.4.

THEOREM 3.10. With the fixed notation of this section let $\operatorname{Rad} B=J$, and let $q_{1}^{*}, \cdots, q_{d}^{*}$ be the prime divisors of zero in the completion $R^{*}$ of $R$. Then there is an integer $n$ (depending on $B$ ) and a nonzero-divisor $c \in\left(B^{n}\right)_{a}$ such that $c A^{\prime}$ has exactly $d$ prime divisors $\mathscr{P}_{i}$ and $R$ is a subspace of $A_{U}^{\prime}$, where $B^{n}=\left(c_{1}, \cdots, c_{e}\right) R$, $A=R\left[c_{1} / c, \cdots, c_{e} / c\right]$, and $U$ is the complement in $A^{\prime}$ of the union of the prime divisors of $c A^{\prime}$. Further,

$$
\operatorname{trd}\left(A^{\prime} / \mathscr{P}_{i}\right) /\left(R / \mathscr{P}_{i} \cap R\right)=\operatorname{depth} q_{i}^{*}-1 .
$$

Proof. Let $\mathscr{R}^{*}=\mathscr{R}\left(R^{*}, B R^{*}\right)$ and let $\mathscr{T}^{*}$ be the total quotient ring of $\mathscr{R}^{*}$. Then $\mathscr{R}^{* \prime}$ is a subring of the completion $W^{*}$ of $W$ (as in the proof of Proposition 2.8). Also, by renumbering if necessary, $Q_{i}^{*}=Q_{i} W^{*} \cap \mathscr{R}^{* \prime} \supset q_{i}^{*} \mathscr{T}^{*} \cap \mathscr{R}^{* \prime}$ and $Q_{i}^{*}$ is a (height one) prime divisor of $u \mathscr{R}^{* \prime}(i=1, \cdots, d)$ (Remark 2.9). Let $U^{*}=\mathscr{R}^{* \prime} \sim$ $\bigcup_{1}^{d} Q_{i}^{*}$ and let $U^{\prime}=\mathscr{R}^{\prime} \sim \bigcup_{1}^{d} Q_{i}$. Then $V^{*}=\mathscr{R}_{U^{*}}^{* \prime}$ is isomorphic to a direct sum of $d$ local Dedekind domains, $V^{*}$ dominates $R^{*}$, and $V^{*} \cap \mathscr{T}=\mathscr{R}_{U^{\prime}}^{\prime}$. Also, since $R^{*}$ is complete, $R^{*}$ is a subspace of $V^{*}$ [2, (30.2)]. Therefore, since $V^{*}$ dominates $\mathscr{R}_{U}^{\prime}$, and $\mathscr{R}_{U^{\prime}}^{\prime}$ dominates $R$, it follows that $R$ is a subspace of $\mathscr{R}_{U^{\prime}}^{\prime}$. Let $n$ be chosen such that there is an element $c \in\left(B^{n}\right)_{a}$ such that $t^{n} c \in Q_{i}$ if and only if $i>d$ (Lemma 3.8), let $B^{n}=\left(c_{1}, \cdots, c_{e}\right) R$, and let $A=R\left[c_{1} / c, \cdots, c_{e} / c\right]$. Then by Lemma $3.4 c A^{\prime}$ has the ideals $\mathscr{P}_{i}=Q_{i} \mathscr{R}^{\prime}\left[1 / t^{n} c\right] \cap A^{\prime}(i=1, \cdots, d)$ as its prime divisors. Let $U=A^{\prime} \sim \bigcup_{1}^{d} \mathscr{P}_{i}$. Then $\mathscr{R}_{U^{\prime}}^{\prime}$ dominates $A_{U}^{\prime}$ and $A_{U}^{\prime}$ dominates $R$, hence $R$ is a subspace of $A_{U}^{\prime}$. The last statement follows from Proposition 2.8 and the Remark following Corollary 3.6 .

4. A characterization of analytically irreducible local domains. Throughout this section $R$ is a local domain with maximal ideal $M$.

THEOREM 4.1. $R$ is analytically irreducible if and only if $R$ is a subspace of a local Dedekind domain. 
Proof. It is well known that a local Dedekind domain is analytically irreducible. Hence, if $R$ is a subspace of such a ring, then $R$ is analytically irreducible. The converse (for altitude $R \geqq 1$ ) follows 3.10. With no restriction on altitude $R$ the converse follows from from Theorem the following.

THEOREM 4.2. Let $R$ be analytically irreducible, let $B$ be an $M$-primary ideal in $R$, let $\mathscr{R}=\mathscr{R}(R, B)$, and let $Q$ be a prime

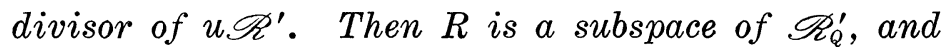

$$
\operatorname{trd}\left(\mathscr{R}^{\prime} / Q\right) /(R / M)=\text { altitude } R \text {. }
$$

Proof. Let $S$ be the complement in $\mathscr{R}^{\prime}$ of the union of the prime divisors of $u \mathscr{R}^{\prime}$. Then $W=\mathscr{R}_{s}^{\prime}$ is a semi-local Dedekind domain and $R$ is a subspace of $W$ (Theorem 2.2). Therefore $\mathscr{R}^{* \prime}$ is a subring of $W^{*} \cap \mathscr{T}^{*}$, where $R^{*}\left(W^{*}\right)$ is the completion of $R(W)$ and $\mathscr{T}^{*}$ is the quotient field of $\mathscr{R}^{*}=\mathscr{R}\left(\mathscr{R}^{*}, B R^{*}\right)$. Let $Q$ be a prime divisor of $u \mathscr{R}^{\prime}$ and let $Q^{*}=Q W^{*} \cap \mathscr{R}^{* \prime}$. Then height $Q^{*}=1$ (Remark 2.9), thus $V^{*}=\mathscr{R}_{Q *}^{* \prime}$ is a local Dedekind domain which contains $R^{*}$ and $N^{*} \cap R^{*}=M R^{*}$, where $N^{*}=Q^{*} V^{*}$. Hence, since $R^{*}$ is complete, $R^{*}$ is a subspace of $V^{*}[2,(30.2)]$. Let $\mathscr{T}$ be the quotient field of $\mathscr{R}$ and let $V=V^{*} \cap \mathscr{T}$. Then $R$ is a subspace of $V$, since $R$ is a subspace of $V^{*}$, and $V=\mathscr{R}_{Q}^{\prime}$, since $N^{*} \cap \mathscr{R}^{\prime}=Q$. The last statement follows since $R$ satisfies the altitude formula [3].

The following Corollary is a special case of Corollary 4.6 below. It is included here because it will be used in the proof of Theorem 4.5 below.

CoROllary 4.3. Let $R$ be analytically irreducible, let altitude $R \geqq 1$, and let $B=\left(b_{1}, \cdots, b_{s}\right) R$ be an $M$-primary ideal. If $b \in B$ is such that $b A^{\prime}$ is a proper ideal, where $A=R\left[b_{1} / b, \cdots, b_{s} / b\right]$, then $R$ $i s$ a subspace of $A_{P}^{\prime}$, for every prime divisor $P$ of $b A^{\prime}$.

Proof. Let $\mathscr{R}=\mathscr{R}(R, B)$ and let $C=\mathscr{R}^{\prime}[1 / t b]$. Then, if $P$ is a prime divisor of $b A^{\prime}$, there is a prime divisor $Q$ of $u \mathscr{R}^{\prime}$ such that $Q C \cap A^{\prime}=P$ (Lemma 3.4). This implies that $\mathscr{R}_{Q}^{\prime}$ dominates $A_{P}^{\prime}$. Hence, since $A_{P}^{\prime}$ dominates $R$ and $R$ is a subspace of $\mathscr{R}_{Q}^{\prime}$ (Theorem 4.2), $R$ is a subspace of $A_{P}^{\prime}$.

REMARK 4.4. Let $R$ be analytically irreducible. (1) $R^{\prime}$ is a local domain. (2) If altitude $R \leqq 1$ and if $(S, N)$ is a local domain that dominates $R$, then $R$ is a subspace of $S$.

Proof. (1) Since $R$ is analytically irreducible, $R^{\prime}$ is a finite $R$ - 
module, and the completion $R^{*}$ of $R$ is a complete local domain. Therefore, the completion $R^{* *}$ of $R^{\prime}$ is $R^{\prime} \otimes_{R} R^{*}[2,(17.8)]$, so $R^{*}=$ $R^{*}\left[R^{\prime}\right]$, hence $R^{*} \leqq R^{*} \cong R^{* \prime}$. Also, $R^{* \prime}$ is a local domain [2, (30.3) and (30.5)], so it follows that $R^{\prime}$ is a local domain. (2) If altitude $R=0$, then $R$ is a field, so $R$ is a subspace of $S$. If altitude $R=1$, then $R^{\prime}$ is a local Dedekind domain (by (1)) which is a finite $R$-module. Thus $A=S\left[R^{\prime}\right]$ is a finite $S$-module, hence $A$ is a semi-local domain that dominates $R^{\prime}$. Therefore $R^{\prime}$ is a subspace of $A$, since the maximal ideal in $R^{\prime}$ is a principal ideal. Since $R$ is a subspace of $R^{\prime}$, it follows that $R$ is a subspace of $S$.

Theorem 4.5 below gives a generalization of Remark 4.4. The following result [10, Lemma 1] is used in the proof of Theorem 4.5: If $x_{1}, \cdots, x_{n}(n>1)$ is a system of parameters in a local domain $(S, N)$, then, with $D=S\left[x_{2} / x_{1}, \cdots, x_{n} / x_{1}\right], N D$ is a height one prime ideal and the $N D$-residues of the $x_{i} / x_{1}$ are algebraically independent over $S / N$.

THEOREM 4.5. $R$ is analytically irreducible if and only if $R$ is a subspace of every local domain $(S, N)$ such that (1) $S$ dominates $R$ and (2) altitude $S+\operatorname{trd}(S / N) /(R / M)=$ altitude $R+\operatorname{trd} S / R<\infty$.

Proof. Let $R$ be analytically irreducible and let $(S, N)$ be a local domain such that (1) and (2) hold. It may be assumed by Remark 4.4 that altitude $R \geqq 2$ (so by (1) altitude $S \geqq 1$ ). If altitude $S=1$, let $V^{*}=S_{P}^{\prime}$ and let $Q^{*}=P V^{*}$, where $P$ is a maximal ideal in $S^{\prime}$. If altitude $S=n>1$, let $x_{1}, \cdots, x_{n}$ be a system of parameters in $S$ and let $D=S\left[x_{2} / x_{1}, \cdots, x_{n} / x_{1}\right]$. Then $N D$ is a height one prime ideal in $D$ and $\operatorname{trd}(D / N D) /(S / N)=n-1$ [10]. Let $V^{*}=D_{P}^{\prime}$ and let $Q^{*}=P V^{*}$, where $P$ is a (height one) prime ideal in $D^{\prime}$ such that $P \cap D=N D$. Then in both cases $\left(V^{*}, Q^{*}\right)$ is a local Dedekind domain [2, (33.10)] which satisfies (1) and (2). Let $T$ be the quotient field of $R$, let $V=V^{*} \cap T$, and let $Q=Q^{*} \cap T$. Then $(V, Q)$ is a local Dedekind domain that dominates $R$. Also,

$$
1+\operatorname{trd}(V / Q) /(R / M) \leqq \text { altitude } R
$$

[11, Prop. 1, p. 330], and, since $Q$ is a principal ideal,

$$
\operatorname{trd}\left(V^{*} / Q^{*}\right) /(V / Q) \leqq \operatorname{trd} V^{*} / V=\operatorname{trd} V^{*} / R<\infty .
$$

Adding these inequalities and using the fact that $\left(V^{*}, Q^{*}\right)$ satisfies (2), it follows that both inequalities are equalities. Therefore $(V, Q)$ also satisfies (2), so there are elements $v_{1}, \cdots, v_{k}$ in $V$ (altitude $R=$ $k+1$ ) such that $V / Q$ is algebraic over $C / Q \cap C$, where $C=R\left[v_{1}, \cdots, v_{k}\right]$. Let $b \in R$ be a common denominator of the $v_{i}$, and let $b V \cap R=B=$ 
$\left(b_{1}, \cdots, b_{s}\right) R$. Then $B$ is $M$-primary, and

$$
C \cong R\left[b_{1} / b, \cdots, b_{s} / b\right]=A \subseteq A^{\prime} \subseteq V .
$$

Therefore $\operatorname{trd}\left(A^{\prime} / Q \cap A^{\prime}\right) /(R / M)=k$, hence, since $R$ satisfies the altitude formula, height $Q \cap A^{\prime}=1$. Thus $V=A_{Q \cap A^{\prime}}^{\prime}$, hence $R$ is a subspace of $V$ (Corollary 4.3). Also $V$ is a subspace of $V^{*}$ (Remark 4.4). Hence, since $V^{*}$ dominates $S$ and $S$ dominates $R, R$ is a subspace of $S$. Conversely, if $R$ is a subspace of every local domain $(S, N)$ which satisfies (1) and (2), then let $x_{1}, \cdots, x_{n}$ be a system of parameters in $R$. It may be assumed that $n=$ altitude $R>0$. Let

$$
D=R\left[x_{2} / x_{1}, \cdots, x_{n} / x_{1}\right] \quad(D=R \text {, if } n=1) .
$$

Then $D^{*}=D_{M D}$ is a local domain of altitude one which satisfies (1) and (2) [10]. Therefore $R$ is a subspace of the local Dedekind domain $D_{P}^{* \prime}$, where $P$ is a maximal ideal in $D^{* \prime}$, hence $R$ is analytically irreducible.

CoRollary 4.6. Let $R$ be analytically irreducible, and let $A$ be an integral domain which is finitely generated over $R$. Then $R$ is a subspace of $A_{P}$, for every prime ideal $P$ in $A$ such that $P \cap R=M$.

Proof. $R$ satisfies the altitude formula [3].

5. A further application. A semi-local ring $R$ is unmixed (quasi-unmixed) in case each prime divisor (each minimal prime divisor) $p$ of zero in the completion of $R$ satisfies depth $p=$ altitude $R$. It is known [3, Prop. 2.2 and Corollary 2.7] that a quasi-unmixed semi-local integral domain satisfies the altitude formula and the second chain condition for prime ideals. It is also known [2, exercise, p. 135] that a pseudo-geometric semi-local domain satisfies the second chain condition for prime ideals if and only if it is unmixed. Theorem 5.2 below contains a form of the converse of the first of these results (since "unmixed" and "quasi-unmixed" are equivalent conditions under the presence of analytical unramifiedness). Also, it is closely related to the second of these results since a pseudo-geometric semi-local domain is analytically unramified [2, (36.4)] and since there is a close relationship between the altitude formula and the second chain condition for prime ideals.

Definition 5.1. A local ring $R$ satisfies the altitude formula in case for each minimal prime ideal $q$ in $R$ it is true that depth $q=$ altitude $R$ and $R / q$ satisfies the altitude formula. A Noetherian ring $A$ satisfies the altitude formula in case $A_{N}$ satisfies the altitude for- 
mula, for each maximal ideal $N$ in $A$.

THEOREM 5.2. Let $(A, N)$ be an analytically unramified local ring. A satisfies the altitude formula if and only if $A$ is unmixed.

Proof. Let $A$ satisfy the altitude formula and let $q$ be a prime divisor of zero in $A$. Then by Lemma 2.3 and hypothesis $R=A / q$ is an analytically unramified local domain which satisfies the altitude formula and altitude $R=$ altitude $A$. Let $M$ be the maximal ideal in $R$, let $B$ be an $M$-primary ideal, let $\mathscr{R}=\mathscr{R}(R, B)$, and let $S$ be the complement in $\mathscr{R}^{\prime}$ of the union of the prime divisors of $u \mathscr{R}^{\prime}$. Then $R$ is a subspace of $W=\mathscr{R}_{S}^{\prime}$ (Theorem 2.2). Let $W^{*}$ be the completion of $W$, and let $p$ be a (minimal) prime divisor of zero in the completion $R^{*}$ of $R$. Then there is a prime divisor $Q$ of $u \mathscr{R}^{\prime}$ such that $p^{*} \cap R^{*}=p$, where $p^{*}$ is the prime divisor of zero in $W^{*}$ which is contained in $Q W^{*}$ (Remark 2.9), and then

$$
\operatorname{depth} p=\operatorname{trd}\left(\mathscr{R}^{\prime} / Q\right) /(R / M)
$$

(Proposition 2.8). Now $\mathscr{R}^{\prime}$ is a finitely generated integral domain over $R$ (Lemma 2.4) and $\operatorname{trd} \mathscr{R}^{\prime} \mid R=$ height $Q=1$, so, since $R$ satisfies the altitude formula, it follows that depth $p=\operatorname{trd}\left(\mathscr{R}^{\prime} / Q\right) /(R / M)=$ altitude $R$. Therefore $R$ is unmixed. Hence $A / q$ is unmixed and depth $q=$ altitude $A$, for each minimal prime ideal $q$ in $A$. Therefore $A$ is quasi-unmixed [3, Lemma 2.2] and analytically unramified, thus $A$ is unmixed. Conversely, let $A$ be unmixed and let $q$ be a minimal prime ideal in $A$. Then $\operatorname{depth} q=$ altitude $A$ and $A / q$ satisfies the altitude formula [3, Lemma 2.2 and Prop. 2.2]. Therefore $A$ satisfies the altitude formula.

CoRollary 5.3. An analytically unramified semi-local ring $R$ satisfies the altitude formula if and only if $R_{M}$ is unmixed, for each maximal ideal $M$ in $R$.

\section{REFERENCES}

1. T. Kikuchi, On the finiteness of the derived normal ring of an affine ring, J. Math. Soc. Japan 15 (1963), 360-365.

2. M. Nagata, Local rings, Interscience Tracts 13, Interscience, New York, 1962.

3. L. J. Ratliff, Jr., On quasi-unmixed semi-local rings and the altitude formula, Amer. J. Math. 87 (1965), 278-284.

4. - Note on analytically unramified semi-local rings, Proc. Amer. Math. Soc. 17 (1966), 274-279.

5. D. Ress, Valuations associated with a local ring (I), Proc. London Math. Soc. 5 (1955), 107-128.

6. — Valuations associated with ideals (I), Proc. London Math. Soc. 6 (1956), 
$161-174$.

7. - Valuations associated with a local ring (II), J. London Math. Soc. 31 (1956), 228-235.

8. - Valuations associated with ideals (II), J. London Math. Soc. 31 (1956), $221-228$.

9. — $A$ note on form rings and ideals, Mathematika 4 (1957), 51-60.

10. H. Sato, On splitting of valuations in extensions of local domains, J. Sci. Hiroshima University 21 (1957), 69-75.

11. O. Zariski and P. Samuel, Commutative Algebra, Volume II, van Nostrand, New York, 1960.

Received September 7, 1967. Work on this paper was supported in part by the National Science Foundation, Grant GP3595 and GP7017.

University of CALifornia, Riverside 



\section{PACIFIC JOURNAL OF MATHEMATICS}

EDITORS

H. ROYDEN

Stanford University

Stanford, California

R. R. Phelps

University of Washington

Seattle, Washington 98105
J. DugundJI

Department of Mathematics

University of Southern California

Los Angeles, California 90007

\section{RICHARD ARENS}

University of California

Los Angeles, California 90024

\section{ASSOCIATE EDITORS}
E. F. BeCKENBACH
B. H. NeUmanN
F. WOLF
K. YOSIDA

\section{SUPPORTING INSTITUTIONS}

UNIVERSITY OF BRITISH COLUMBIA CALIFORNIA INSTITUTE OF TECHNOLOGY UNIVERSITY OF CALIFORNIA MONTANA STATE UNIVERSITY UNIVERSITY OF NEVADA NEW MEXICO STATE UNIVERSITY OREGON STATE UNIVERSITY UNIVERSITY OF OREGON OSAKA UNIVERSITY UNIVERSITY OF SOUTHERN CALIFORNIA
STANFORD UNIVERSITY UNIVERSITY OF TOKYO UNIVERSITY OF UTAH WASHINGTON STATE UNIVERSITY UNIVERSITY OF WASHINGTON

AMERICAN MATHEMATICAL SOCIETY CHEVRON RESEARCH CORPORATION TRW SYSTEMS NAVAL WEAPONS CENTER 


\section{Pacific Journal of Mathematics \\ Vol. 27, No. $1 \quad$ January, 1968}

Willard Ellis Baxter, On rings with proper involution ............... 1

Donald John Charles Bures, Tensor products of $W^{*}$-algebras........... 13

James Calvert, Integral inequalities involving second order derivatives . . . . 39

Edward Dewey Davis, Further remarks on ideals of the principal class.... 49

Le Baron O. Ferguson, Uniform approximation by polynomials with integral

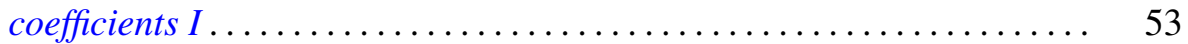

Francis James Flanigan, Algebraic geography: Varieties of structure

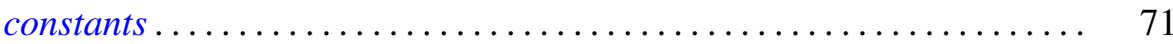

Denis Ragan Floyd, On QF -1 algebras ..................... 81

David Scott Geiger, Closed systems of functions and predicates ......... 95

Delma Joseph Hebert, Jr. and Howard E. Lacey, On supports of regular Borel measures ................................... 101

Martin Edward Price, On the variation of the Bernstein polynomials of a function of unbounded variation ........................ 119

Louise Arakelian Raphael, On a characterization of infinite complex matrices mapping the space of analytic sequences into itself........ 123

Louis Jackson Ratliff, Jr., A characterization of analytically unramified

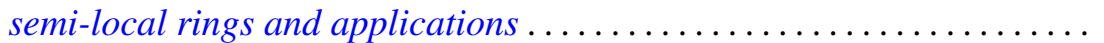

S. A. E. Sherif, A Tauberian relation between the Borel and the Lototsky transforms of series ................................ 145

Robert C. Sine, Geometric theory of a single Markov operator .......... 155

Armond E. Spencer, Maximal nonnormal chains in finite groups......... 167

Li Pi Su, Algebraic properties of certain rings of continuous functions .... 175

G. P. Szegô, A theorem of Rolle's type in $E^{n}$ for functions of the class $C^{1} \ldots 193$

Giovanni Viglino, A co-topological application to minimal spaces ........ 197

B. R. Wenner, Dimension on boundaries of $\varepsilon$-spheres ............... 201 